\title{
Globally Standardized MODIS Spectral Mixture Models
}

\author{
Daniel Sousa* and Christopher Small \\ Lamont-Doherty Earth Observatory, Columbia University, Palisades, NY \\ *Corresponding Author: Daniel Sousa (d.sousa@columbia.edu)
}

\begin{abstract}
This analysis presents standardized substrate, vegetation, and dark (S, V, \& D) endmembers (EMs) for spectral mixture analysis (SMA) of MODIS daily nadir-looking BRDF-adjusted reflectance (daily NBAR) data. MODIS daily NBAR EMs are derived from a diverse collection of over 43 million MODIS spectra spanning 6 continents and all non-polar biomes. EM spectra found in the study are comparable to those of previous studies using decameter Landsat and Sentinel imagery. Sensitivity analysis of SVD mixture models based on 351 pairs of 27 possible single-pixel EM combinations shows mean S, V, and D fraction estimates to differ by $4 \pm 3 \%, 3$ $\pm 2 \%$, and $3 \pm 2 \%$, respectively. In addition to the SVD EMs, an additional snow EM is also identified. This snow EM is deemed tentative pending a more detailed analysis of cryospheric environments. Vicarious validation based on unmixing of coincident Landsat 8 spectra shows complementary -7 and $+11 \%$ biases in S and D EM fractions, but less than $0.5 \%$ bias in V EM fractions. Similar 6 to 7\% dispersion in Landsat vs MODIS estimates is observed for all three fractions. Model misfit for the 4-EM SVD+snow MODIS model is low (> 99.9\% of pixels with RMSE < 5\%). V fraction is compared to Normalized Difference, Enhanced and Soil Adjusted Vegetation Indices (NDVI, EVI and SAVI). NDVI has over 2x the dispersion (29\% vs 13 to $14 \%)$ and over $4 x$ the bias (+18\% vs $4 \%$ ) of EVI and SAVI when compared against V fraction. Combined with previous studies, these results extend the scaling linearity and low misfit of the global SVD model from $2 \mathrm{~m}$ up to $500 \mathrm{~m}$.
\end{abstract}

Keywords

MODIS daily NBAR, linear spectral mixture, Landsat, scaling

\section{Introduction}

Spectral mixture analysis (SMA) is a robust, physically-based method of continuous land cover mapping from multispectral optical imagery [1-3]. SMA represents the aggregate reflectance of a mixed pixel as a linear mixture of the areal abundance of its constituent endmember (EM) reflectances. EM fraction estimates scale linearly, even in multiple scattering landscapes such as cities [4] and deserts [5]. Using either field-based [6] or vicarious (multiresolution) [7,8] validation techniques, SMA generally outperforms spectral indices in terms of both comparison with in situ observations and scaling linearity. These reasons, combined with its physical rigor, conceptual simplicity, and ease of implementation make SMA the method of choice for a wide range of mapping applications. For an excellent introduction to SMA, see [9].

For some applications, a potential limitation of SMA is its reliance on scene-specific EM reflectance spectra. However, the global analysis of [10] showed that the decameter multispectral 
reflectance of a wide range of landscapes can be accurately represented using a generic 3-EM spectral mixture model based on rock and soil Substrate, illuminated photosynthetic Vegetation, and Dark targets like water and shadow (S, V, and D). SVD EMs spanning a spectrally diverse mixing space can accurately represent the full range of ternary spectral mixtures bounded by the EMs. As the diversity of the mixing space approaches global diversity, the utility of standardized bounding EMs expands accordingly. Spectrally distinct EMs not included in the SVD model include snow, evaporite deposits, and coral reefs and shallow marine substrates, although they can be included when required. The SVD model has been corroborated and extended by subsequent studies to include diverse global collections spanning Landsat 4/5/7/8 [8,11] and Sentinel-2 [12] sensors, as well as a spatially limited but spectrally diverse regional collection of hyperspectral AVIRIS flight lines [13]. To our knowledge, however, no comparable analysis has extended this work to include the 18+ year near-daily MODIS satellite archive.

A number of complications (e.g. [14-17]) are significantly more severe in MODIS data than in imagery from near-nadir looking decameter resolution sensors like those on the Landsats and Sentinel-2. Attempting to obtain global MODIS EMs without comprehensively accounting for these issues would be futile because artifacts caused by variations in pixel footprint, sun-sensor geometry, topography and BRDF could overprint real spectral differences among materials. However, the recently operationalized daily nadir view BRDF-adjusted reflectance (NBAR) product [18] is designed to minimize these complications and provide an optimized surface reflectance product for time series terrestrial monitoring. The standardized NBAR product is a significant advance over previous MODIS processing schemes and allows for straightforward cross-comparison of land surface reflectance between MODIS acquisitions. Operationalization of the NBAR product facilitates the identification of MODIS-specific global EMs. This extends the range of potential applications for multitemporal SMA to include larger synoptic areas with more frequent revisit than Landsat and a 15-year deeper historical archive than Sentinel 2.

This analysis presents standardized global MODIS EMs determined from daily NBAR imagery of 9 cloud-free MODIS tiles spanning 6 continents and nearly all non-polar terrestrial biomes. Using similar methodology to $[8,10,11]$, we characterize the dimensionality and topology of the MODIS spectral feature space and compare it to previous decameter-resolution studies. We also conduct a scaling comparison between coincident $30 \mathrm{~m}$ Landsat 8 and $500 \mathrm{~m}$ MODIS daily NBAR EM fractions. The EMs presented here will enable estimation of globally consistent EM fraction maps throughout the MODIS archive for retrospective analysis and prospective monitoring of changes in the Earth surface.

\section{Methods}

Data were downloaded from the NASA Earthdata website (https://search.earthdata.nasa.gov/). Tiles were chosen on the basis of spectral diversity and minimum atmospheric contamination. Daily NBAR tiles were composited into a single image and the covariance-based principal component (PC) transform was applied. The correlation-based transform was also investigated, but the resulting feature space was less informative. Feature space topology was investigated and EMs were selected interactively. The methodology and rationale for EM selection used here is explained in more detail in $[8,11]$. 


\section{Analysis}

The 9 MODIS tile locations were chosen on the basis of spectral and land cover diversity (Fig. 1 and 2). These regions were selected in order to span a wide range of natural and anthropogenic land cover. The full range of non-polar biomes and elevations was included. While tropical forests do not account for a large fraction of the study area, the locations included span large biodiversity gradients so the spectral diversity is disproportionate. Also, previous experience with global multispectral image analysis suggests that closed canopy forests are generally wellrepresented as binary mixtures of $\mathrm{V}$ and $\mathrm{D}$ fractions and unlikely to host global vegetation EMs at pixel scales. Scenes were selected to include a particularly wide range of lithologic and soil diversity, including representative mafic, felsic, and intermediate crystalline bedrock; alluvium, colluvium, and aeolian sediment of variable grain size and composition; and soils of a wide range of ages, compositions and origins. The scale of the MODIS GIFOV was too coarse to resolve specific anthropogenic building materials and impervious surfaces.

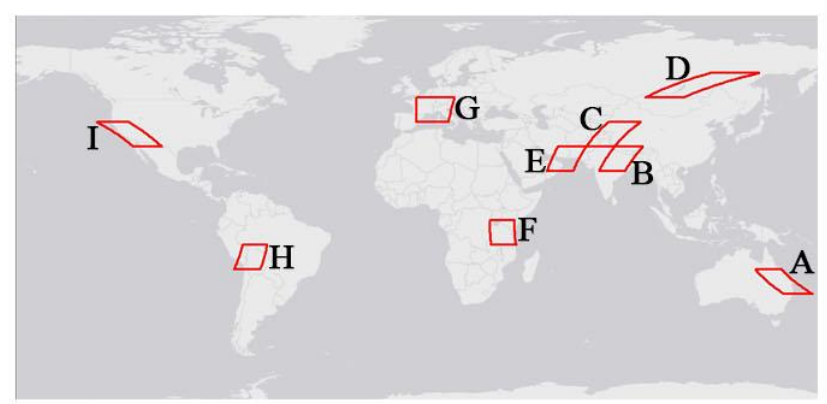

Figure 1. Locations of the 9 MODIS Daily NBAR tiles used in this analysis. A wide range of spectral diversity is present, including tropical and boreal forests, agriculture, deserts, mountains with snow and ice, turbid and clear water (both deep and shallow), evaporite minerals and reefs. Labels A-I correspond to image labels in Figure 2. Subscene I (California) was used for the Landsat scaling comparison shown in Figure 6.
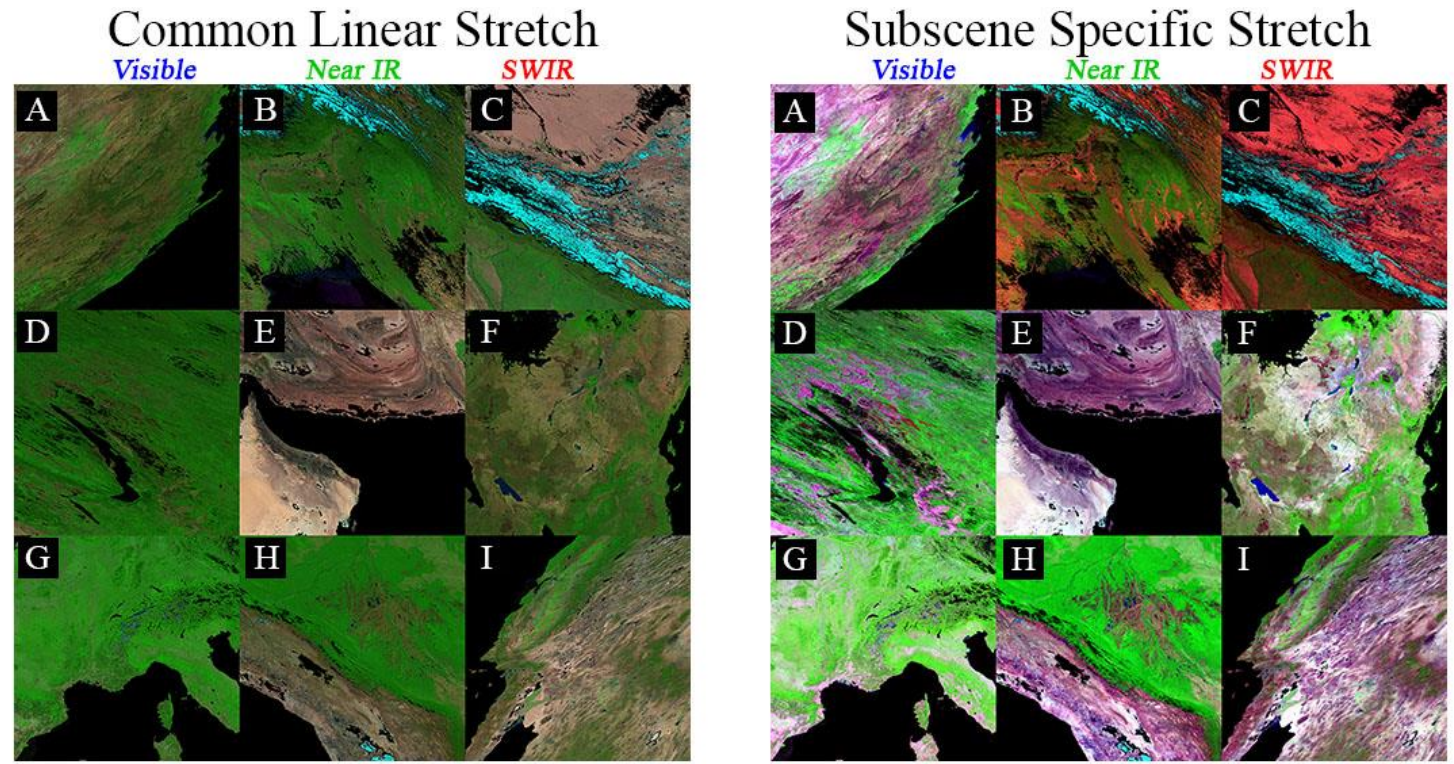

Figure 2. Comparison of 9 MODIS Daily NBAR tiles from the locations shown in Figure 1. Each tile is shown in Sinusoidal projection with both a common linear stretch (reflectance $=0$ to 0.7 ) as well as with subscene-specific $2 \%$ linear stretches to illustrate the spectral diversity of the locations chosen. The tiles sample a range of evergreen and deciduous natural vegetation, agriculture, and standing water (both deep and shallow), as well as lithologically variable soil, sediment, and rock substrates. With the exception of the snow in B \& $\mathrm{C}$ and evaporites in $\mathrm{H} \& \mathrm{I}$, all tiles are composed of varying mixtures of rock and soil substrates, vegetation, water and shadow. 
Spectral characterization of the global daily NBAR dataset is shown in Fig. 3 and 4. Sensitivity of feature space dimensionality and topology was examined by comparison of results from analysis of the full 9 tile dataset to results obtained by grouping the dataset into 3 subsets of 3 tiles (shown as red, green and blue on Fig. 3). Separate individual analysis of each tile was also conducted (shown as gray on Fig. 3). As expected, the individual tiles and 3-tile subsets with appreciable amounts of snow cover (B \& C; blue subset), and evaporite deposits (H \& I; green subset) show marginally higher variance in dimensions 3-7 than the tiles and subset without these features. The dimensionality of the full dataset is an aggregate of the subsets, and the break between dimensions 3 and 4 is clearest in the full dataset. Despite these differences, > 99\% of variance is present in the first 3 dimensions of the full dataset and every 3-tile subset, and $>93 \%$ is present in the first three dimensions of each individual tile.

The SVD ternary mixing space structure is evident in each of the 3-tile spatial subsets (not shown), as well as full 9-tile composite (Fig. 4). Spectral consistency of pixels near the apexes of the mixing space indicates that EM variability is even less pronounced than in global Landsat mixing spaces. Global MODIS daily NBAR S, V, and D EMs are shown in the lower right (thick lines), alongside EMs from the 3-tile subsets (thin lines). EM sensitivity analysis was conducted by unmixing the full 9-tile dataset using all 27 possible combinations of the 3- and 9-tile EMs. Fraction difference images were computed for all 351 possible pairs of these 27 mixture models. EM sensitivity was greatest for the $\mathrm{S}$ fraction, with 4\% mean, 3\% standard deviation, and 9\% maximum image-to-image biases between sets of EMs. Both V and D fractions showed 3\% mean, $2 \%$ standard deviation, and $9 \%$ maximum biases.

Deviations from the primary SVD ternary mixing space were due to the spatially extensive snow cover in the Himalaya tiles and evaporite deposits in the Atacama Desert. Because of the prominence of the snow limb, an additional snow EM was selected. However, we recommend this EM be used with caution due to the complex optical properties of snow. The values the global MODIS SVD EMs, as well as the tentative snow EM, are given in Table 1.

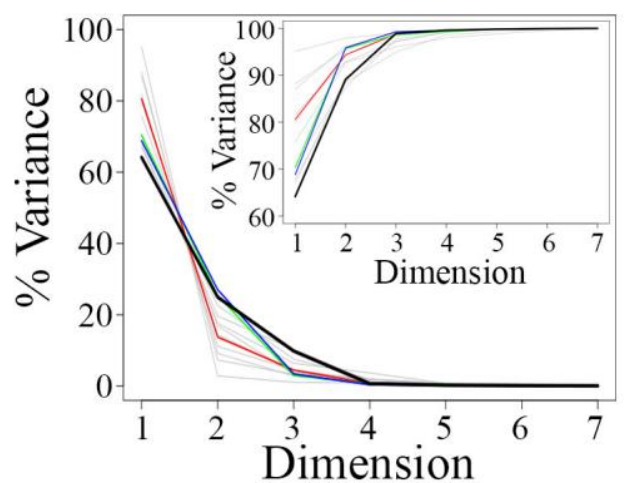

Figure 3. Partition of variance. Both dimension-specific (main plot) and cumulative (inset) results are shown. The full 9-tile dataset (black) is decomposed into 3 subsets of 3 tiles each (red, green, blue) as well as each individual tile (gray). One of the subsets (blue) contains all tiles with snow and ice.Another subset (green) contains several pixels with evaporite minearls. The fact that these pixels lie outside the primary mixing space results in higher variance for PC 2 than the other subset (red) with only SVD pixels. These pixels are carried over into the global dataset, resulting in elevated variance in PC 2 for that rotation as well. The spectral signature characterized by EOF 2 of the red subset is thus pushed into the 3rd dimension of the global set. Even with the snow and evaporite pixels, $>99 \%$ of the variance for the global set of tiles is contained in the first 3 dimensions. 

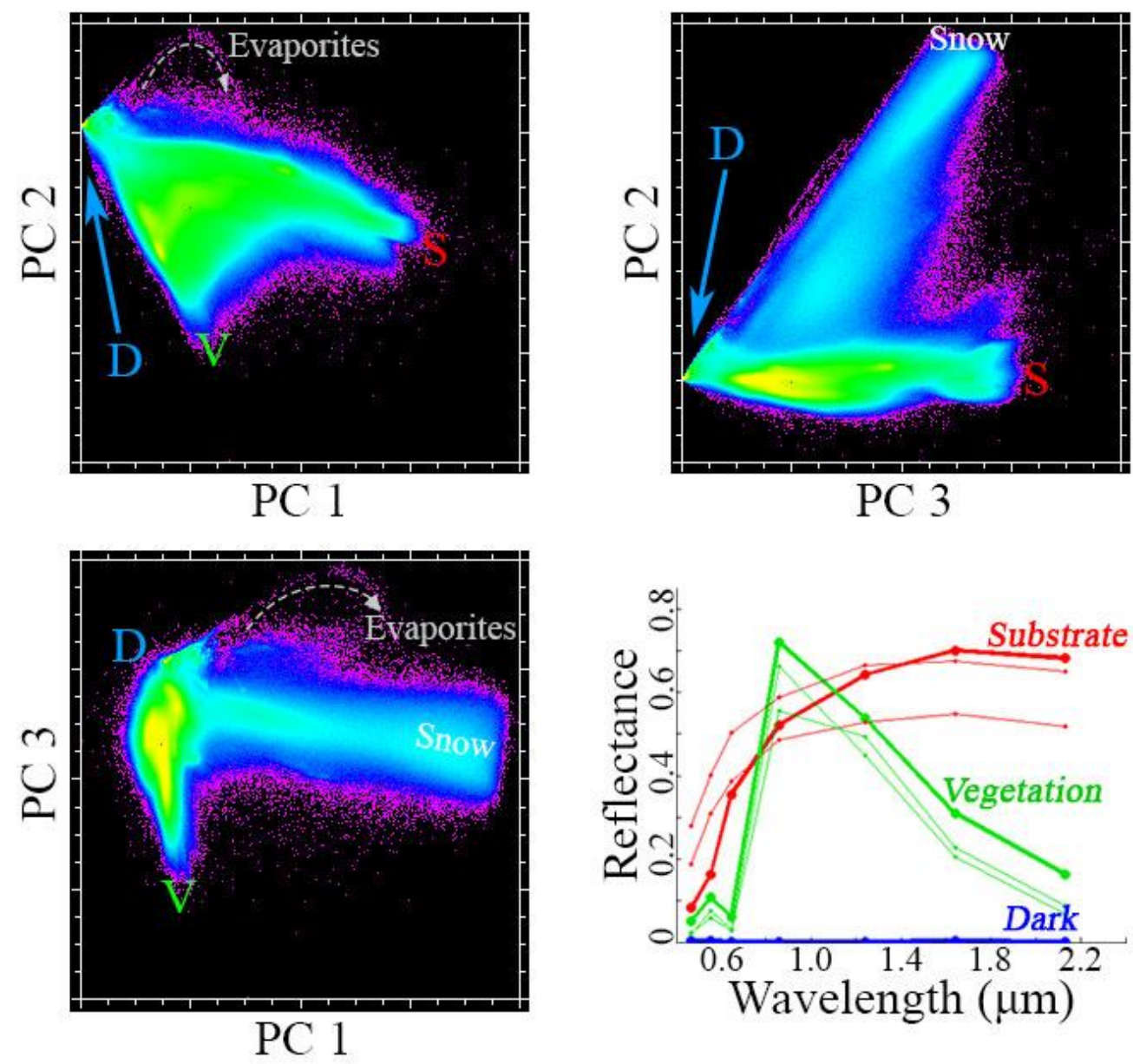

Figure 4. The MODIS Daily NBAR spectral mixing space derived from 43,131,344 broadband spectra. The Landsat mixing space (not shown) is characterized by the same geometric structure. Endmember (EM) spectra selected from the apexes of the scatterplot correspond to the most extreme pixels of each land cover type. EMs are chosen from three different subsets of 3 tiles (thin) as well as for the 9 tile global composite. In each case, subset feature spaces (not shown) are similar to the global feature space. Each global EM is also one of the subset EMs. The prominent cluster protuding out from the SVD mixing space represents the Snow in the tiles containing the Himalaya. The small but coherent nonlinear mixing curve emerging from the Dark apex corresponds to evaporite pans in the Atacama \& Mojave Deserts.

\begin{tabular}{|c|c|c|c|c|}
\hline $\begin{array}{c}\text { Wavelength } \\
(\boldsymbol{\mu m})\end{array}$ & $\begin{array}{c}\text { Substrate EM } \\
\text { Reflectance }\end{array}$ & $\begin{array}{c}\text { Vegetation EM } \\
\text { Reflectance }\end{array}$ & $\begin{array}{c}\text { Dark EM } \\
\text { Reflectance }\end{array}$ & $\begin{array}{c}\text { Snow EM } \\
\text { Reflectance }\end{array}$ \\
\hline $\mathbf{0 . 4 7}$ & 0.0835 & 0.0515 & 0.0042 & 0.9268 \\
\hline $\mathbf{0 . 5 6}$ & 0.1639 & 0.1073 & 0.0041 & 0.9611 \\
\hline $\mathbf{0 . 6 5}$ & 0.3539 & 0.0600 & 0.0004 & 0.9699 \\
\hline $\mathbf{0 . 8 6}$ & 0.5204 & 0.7204 & 0.0001 & 0.9295 \\
\hline $\mathbf{1 . 2 4}$ & 0.6413 & 0.5374 & 0.0001 & 0.5425 \\
\hline $\mathbf{1 . 6 4}$ & 0.6998 & 0.3098 & 0.0027 & 0.1146 \\
\hline $\mathbf{2 . 1 3}$ & 0.6811 & 0.1631 & 0.0005 & 0.0437 \\
\hline
\end{tabular}

Table 1. Reflectance values for global MODIS Substrate, Vegetation, Dark (SVD) \& Snow EMs. 

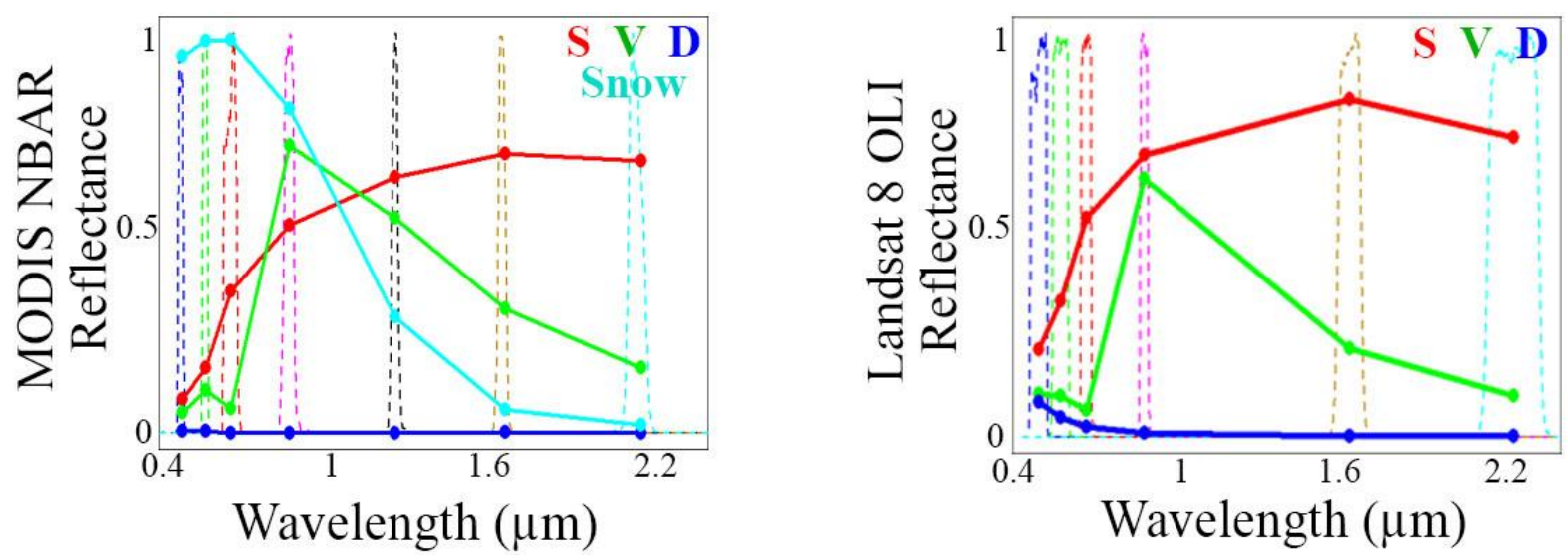

Figure 5. Comparison of MODIS and Landsat 8 OLI global EMs and spectral response functions. The MODIS sensor features an extra NIR band, as well as substantially narrower SWIR bands compared to OLI. The MODIS global Substrate EM is substantially darker than the the OLI EM across all wavelengths. The MODIS global Vegetation and Dark EMs are both darker in the visible than the OLI EMs as a result of the removal of Rayleigh scatering by the NBAR model. A tentative snow MODIS EM is also identified.

Fig. 5 shows the global EMs from this analysis in comparison to those from the recent Landsat $7 / 8$ cross calibration analysis of [11]. As expected, the MODIS S EM is substantially darker across all wavelengths than the Landsat S EM (mean band difference $=0.15, \sigma=0.05$ ). In contrast, the MODIS V EM is brighter across most wavelengths than the Landsat V EM (mean band difference $=0.03, \sigma=0.05$ ), with a somewhat steeper red edge (NIR - Red difference 0.66 vs. 0.57). The NBAR atmospheric correction is evident in the reduced/eliminated Rayleigh scattering profile at visible wavelengths. However, despite the differences in number of spectral bands, spectral bandpass width, and spatial resolution, the MODIS and Landsat feature spaces and S, V, D EMs are remarkably similar. Apparently, sufficient spectrally homogenous regions are present globally to allow for identification of representative EMs at $500 \mathrm{~m}$ resolution that coincide with those of $30 \mathrm{~m}$ resolution derived from much more globally extensive sampling.

Cross-sensor scaling of fraction estimates was compared using Landsat 8 imagery coincident to a portion of the California MODIS tile (Path 42, Rows 33 through 36, 2016 JD 229). The degree of spectral diversity present in California [13] suggests this subset can be used as an approximately representative sample of the global dataset. The Landsat tiles, comprised of 96,829,558 usable pixels, were mosaiced, masked, and convolved to $500 \mathrm{~m}$ resolution using a low pass Gaussian filter scaled to match the MODIS point spread function, then unmixed using global SVD EMs from [11]. After processing and resampling to the $500 \mathrm{~m}$ MODIS grid, 348,568 pixels remained for direct comparison with the MODIS fractions. MODIS data were unmixed using both 3(SVD) and 4- (SVD+snow) EM mixture models. Least squares inversion of each mixture model included an equal weight (1.0) unit sum constraint. Resulting fraction estimates spanned the [0,1] range with no estimates $>1$ or significantly $<0$.

Fraction differences between 3- and 4-EM models were small for S $(0.01 \pm 0.01), \mathrm{V}(0.02 \pm$ $0.02)$, and $\mathrm{D}(0.01 \% \pm 0.01 \%)$. However, misfit was substantially higher for the 3 -EM model (95\% pixels with RMSE < 5\%) than the 4-EM model (>99.9\% pixels with RMSE < 5\%). This can be explained by poor 3-EM model misfit for pixels with partial or complete ice and snow cover in the Sierra Nevada. The 4-EM model also slightly outperformed the 3-EM Landsat model $(>99.8 \%$ pixels with RMSE $<5 \%)$. 
Fig. 6 compares S, V, and D fraction estimates for the California subset estimated using the 4EM MODIS model and the 3-EM Landsat model. Complementary biases of $-7 \%$ and $+11 \%$ were observed in S and D fractions, respectively. This is expected given the difference in albedo between the Landsat and MODIS S EMs, as the Landsat EM is derived from pure sand. For most applications a high albedo soil is more representative than sand for the substrate EM. If required, this bias is easily correctable with a simple linear transformation. V fraction also scales linearly, showing less than $0.5 \%$ bias. All fractions show comparable dispersion ( $\sigma \approx 6$ to $7 \%$ ), likely due to subpixel displacements and/or variations in MODIS observational coverage.
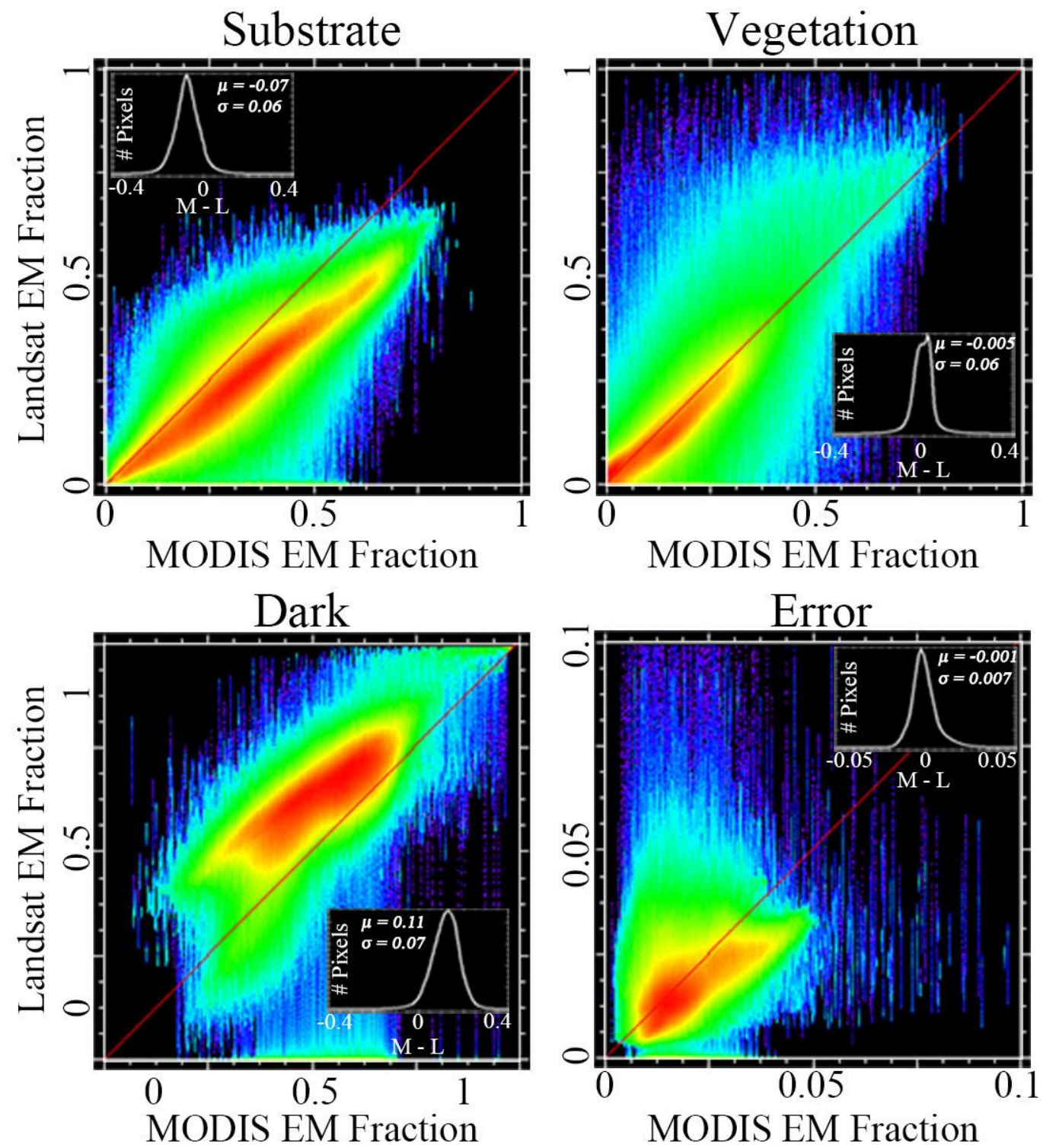

Figure 6. Scaling comparison for SVD fractions derived from 348,568 same-day MODIS Daily NBAR and 96,829,558 Landsat 8 OLI spectra using the new global Daily NBAR SVD+I EMs and the Landsat 7/8 underflight SVD EMs from Sousa \& Small, 2017. Vegetation fraction is linear with negligible bias $(\mu<-0.5 \%)$. Substrate and dark fractions are also linear. Positive bias in the Dark fraction offsets negative bias in the substrate fraction, expected given the brighter Landsat Substrate EM. Modest dispersion $(\sigma=6$ to $7 \%$ ) is observed in all fractions, likely due to subpixel displacements or variations in MODIS observational coverage. Inset histograms show Modis - Landsat fraction difference distributions. Misfit esimates for both sensors are comparable, with $>99.8 \%$ of all pixels with $<5 \%$ RMSE in each case. Unmixing MODIS without the I EM yields similar results for S, V, and D fractions, but with higher misfits ( $99 \%$ of pixels $<10 \%$ RMSE). 
Finally, SMA-derived V fraction is compared to commonly used spectral vegetation indices for all 43,131,344 MODIS spectra (Fig. 7). The Enhanced Vegetation Index (EVI, [19]) and Soil Adjusted Vegetation Index (SAVI, [20]) agree with V fraction remarkably well, each showing a $4 \%$ bias and 13 to $14 \%$ dispersion. In contrast, the Normalized Difference Vegetation Index (NDVI, [21]) shows much greater dispersion, slope, and curvature while beginning to saturate at fractions $<0.5$. NDVI substantially overestimates $\mathrm{V}$ fraction, with over $4 \mathrm{x}$ greater bias $(18 \%)$ and $2 x$ greater dispersion $(29 \%)$ than either EVI or SAVI.
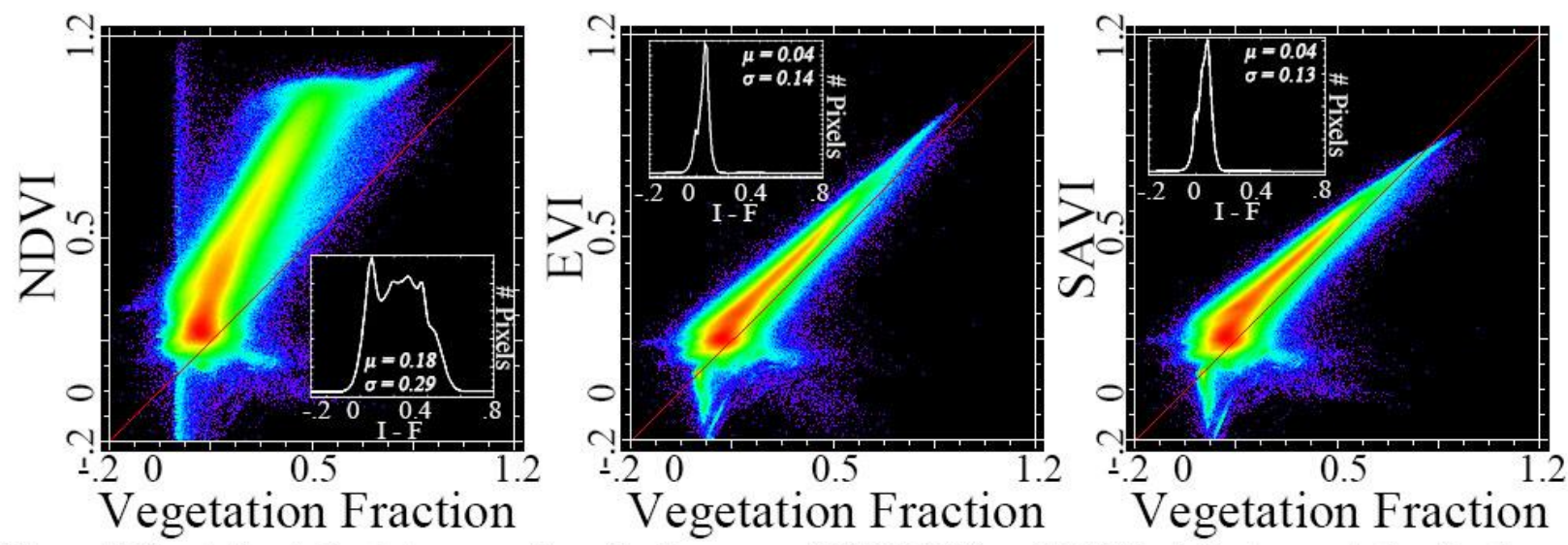

Figure 7. Vegetation index intercomparison. Performance of NDVI, EVI, and SAVI relative to vegetation fraction for MODIS Daily NBAR. Inset histograms show Index - Fraction differences. EVI and SAVI both show a generally linear relationship to vegetation fraction with minor overestimation $(\mu=0.04)$ and dispersion $(\sigma=0.14)$. NDVI overestimates more severely across all fractions $(\mu=0.18)$ with greater dispersion $(\sigma=0.29)$.

\section{Discussion and Conclusion}

This study extends previous decameter-scale global analyses of spectral characterization and global EM identification to include hectometer-scale observations from the MODIS daily NBAR product. Using a spectrally and geographically diverse set of images, we find that the MODIS spectral feature space possesses the same SVD ternary linear mixing properties as the Landsat, Sentinel-2 and AVIRIS mixing spaces. This degree of similarity is significant given the differences in spatial and spectral resolution among sensors. The global EMs found in this study can be used as the basis of a standardized approach to rigorous land cover fraction estimation from the daily NBAR product throughout the 18+ year MODIS archive.

The $8 \%$ dispersion in the Landsat vs MODIS comparison indicates the degree to which the processing routines applied to generate the NBAR product are able to overcome MODIS data complexities such as geolocation, BRDF and terrain correction, relative to the frame of reference of a coincident Landsat acquisition. The $\mathrm{S}$ and $\mathrm{D}$ fraction biases are expected given the difference in S EM albedo and can be easily corrected using a simple linear transformation.

Standardized global EMs minimize the site specificity of SMA resulting from the use of imagespecific EMs. By using a common set of basis vectors, rigorous subpixel areal abundance can be estimated with trivial computational cost and compared directly across spatial and temporal domains. These estimates scale linearly, a key advantage over normalized difference spectral indices for multiscale analyses. The linear scaling and $<0.5 \% \mathrm{~V}$ fraction bias between MODIS and Landsat suggest considerable potential for global Landsat \& MODIS EMs to be used together in studies requiring multisensor fusion. 
Despite the strength of the standardized approach, however, we also strongly recommend the independent examination of the spectral feature space for each new study area. For many applications local spectral EMs may be more appropriate, but including global EM fraction estimation may provide additional context for global EMs. Given our experience with the variability of multispectral EMs, we feel this is particularly important for the S EM because the sands that occupy this apex of the mixing space are much brighter than most soils. Furthermore, while this analysis was designed to be as comprehensive as possible, it is not exhaustive. It is possible that a particular study area may contain an even more extreme S EM than found here. More likely, however, is that the S EM in a particular area has a less extreme (i.e. lower albedo) reflectance signature than that found in this global compilation. In this case, using a local S EM is likely to produce more accurate $\mathrm{S}$ and $\mathrm{D}$ fraction estimates than using the global S EM. This can also generally be corrected for using a simple linear transformation from global EM estimates. Finally, it is important to note that for SMA to be applied successfully to data from other wide-field sensors, similarly rigorous preprocessing will likely need to be implemented before imaging artifacts are suppressed to the level that the advantages of SMA will manifest.

\section{Acknowledgements}

This analysis would not have been possible without the extensive work done by the MODIS team develop and implement preprocessing of MODIS data to daily NBAR specifications. Work done by DS was conducted with Government support under FA9550-11-C-0028 and awarded by the Department of Defense, Air Force Office of Scientific Research, National Defense Science and Engineering Graduate (NDSEG) Fellowship, 32 CFR 168a. CS acknowledges the support of the NASA MultiSensor Land Imaging program (grant NNX15AT65G).

\section{References}

1. Adams, J.B.; Smith, M.O.; Johnson, P.E. Spectral mixture modeling: A new analysis of rock and soil types at the Viking Lander 1 site. J. Geophys. Res. Solid Earth 1986, 91, 8098-8112, doi:0.1029/JB091iB08p08098.

2. Smith, M.O.; Johnson, P.E.; Adams, J.B. Quantitative determination of mineral types and abundances from reflectance spectra using principal components analysis. J. Geophys. Res. Solid Earth 1985, 90, C797-C804, doi:10.1029/JB090iS02p0C797.

3. Gillespie, A.R.; Smith, M.O.; Adams, J.B.; Willis, S.C.; Fischer, A.F.; Sabol, D.E. Interpretation of residual images: spectral mixture analysis of AVIRIS images, Owens Valley, California. In Annual JPL Airborne Visible/Infrared Imaging Spectrometer (AVIRIS) Workshop; 1990; Vol. 2, pp. 54-90.

4. Small, C. Estimation of urban vegetation abundance by spectral mixture analysis. Int. J. Remote Sens. 2001, 22, 1305-1334, doi:10.1080/01431160151144369.

5. Smith, M.O.; Ustin, S.L.; Adams, J.B.; Gillespie, A.R. Vegetation in deserts. I. A regional measure of abundance from multispectral images. Remote Sens. Environ. 1990, 31, 1-26.

6. Elmore, A.J.; Mustard, J.F.; Manning, S.J.; Lobell, D.B. Quantifying vegetation change in semiarid environments: precision and accuracy of spectral mixture analysis and the normalized difference vegetation index. Remote Sens. Environ. 2000, 73, 87-102.

7. Small, C.; Lu, J.W.T. Estimation and vicarious validation of urban vegetation abundance 
by spectral mixture analysis. Remote Sens. Environ. 2006, 100, 441-456, doi:10.1016/J.RSE.2005.10.023.

8. Small, C.; Milesi, C. Multi-scale standardized spectral mixture models. Remote Sens. Environ. 2013, 136, 442-454, doi:10.1016/j.rse.2013.05.024.

9. Adams, J.B.; Gillespie, A.R. Remote Sensing of Landscapes with Spectral Images; Cambridge University Press: New York, 2006; ISBN 978-0-521-6622-1-5.

10. Small, C. The Landsat ETM+ spectral mixing space. Remote Sens. Environ. 2004, 93, 117, doi:10.1016/j.rse.2004.06.007.

11. Sousa, D.; Small, C. Global cross-calibration of Landsat spectral mixture models. Remote Sens. Environ. 2017, 192, doi:10.1016/j.rse.2017.01.033.

12. Small, C. Multisource imaging of urban growth and infrastructure using Landsat, Sentinel and SRTM. In NASA Landsat-Sentinel Science Team Meeting; Rockville, MD, 2018.

13. Sousa, D.; Small, C. Multisensor analysis of spectral dimensionality and soil diversity in the great central valley of California. Sensors (Switzerland) 2018, 18.

14. Tan, B.; Woodcock, C.; Hu, J; Zhang, P.; Ozdogan, M.; Huang, D.; Yang, W.;

Knyazikhin, Y.; Myneni, R. The impact of gridding artifacts on the local spatial properties of MODIS data: Implications for validation, compositing, and band-to-band registration across resolutions. Remote Sens. Environ. 2006, 105, 98-114, doi:10.1016/j.rse.2006.06.008.

15. Fensholt, R.; Sandholt, I.; Proud, S.; Stisen, S.; Rasmussen, M. Assessment of MODIS sun-sensor geometry variations effect on observed NDVI using MSG SEVIRI geostationary data. Int. J. Remote Sens. 2010, 31, 6163-6187, doi:10.1080/01431160903401387.

16. Xin, Q.; Woodcock, C.; Liu, J.; Tan, B.; Mellow, R.; Davis, R. View angle effects on MODIS snow mapping in forests. Remote Sens. Environ. 2012, 118, 50-59.

17. Sims, D.; Rahman, A.; Vermote, E.; Jiang, Z. Seasonal and inter-annual variation in view angle effects on MODIS vegetation indices at three forest sites. Remote Sens. Environ. 2011, 115, 3112-3120, doi:10.1016/j.rse.2011.06.018.

18. Ju, J.; Roy, D.P.; Shuai, Y.; Schaaf, C. Development of an approach for generation of temporally complete daily nadir MODIS reflectance time series. Remote Sens. Environ. 2010, 114, 1-20, doi:10.1016/j.rse.2009.05.022.

19. Huete, A.; Didan, K.; Miura, T.; Rodriguez, E.P.; Gao, X.; Ferreira, L.G. Overview of the radiometric and biophysical performance of the MODIS vegetation indices. Remote Sens. Environ. 2002, 83, 195-213, doi:http://dx.doi.org/10.1016/S0034-4257(02)00096-2.

20. Huete, A.. A soil-adjusted vegetation index (SAVI). Remote Sens. Environ. 1988, 25, 295-309, doi:10.1016/0034-4257(88)90106-X.

21. Rouse, J.W.. J.; Haas, R.H.; Schell, J.A.; Deering, D.W. Monitoring vegetation systems in the Great Plains with ERTS, in Third Earth Resources Technology Satellite-1 Symposium. NASA SP-351., edited by S. C. Freden, E. P. Mercanti, and M. A. Becker, 1974, p. 309, NASA, Washington, D.C. 